Les nouvelles

de ce numéro

ont été préparées par

J.-C. Dreyfus

A. Kahn

J.-P. Grünfeld

\section{Gènes paternels et maternels." différence de gravité des maladies héréditaires}

Certaines maladies humaines héréditaires à transmission autosomique dominante ont une gravité différente selon qu'elles sont héritées du père ou de la mère. Dans une nouvelle $\left(m / s n^{\circ} 6 p\right.$. 336) nous avons rapporté des arguments expérimentaux montrant que chez la souris certains chromosomes autosomes avaient des propriétés différentes chez le mâle et chez la femelle. Peut-il en être de même en pathologie?

On connaît au moins trois maladies à hérédité dominante autosomique qui, à des degrés variables, entrent dans cette catégorie. La plus étudiée est la chorée de Huntington, dont le gène est porté par le chromosome 4. Des centaines de familles ont été passées en revue; il apparaît clairement que les formes précoces (se manifestant avant 20 ans) sont le plus souvent transmises par le père, alors que les formes d'apparition tardive (après 50 ans) le sont surtout par la mère $[I, 2]$. Cette notion est confirmée par toutes les statistiques chez les Blancs; elle n'a toutefois peut-être pas valeur universelle, puisqu'une étude limitée portant sur des Noirs américains semble aboutir à des résultats inverses (cinq formes juvéniles d'origine maternelle sur six) [3]. On peut d'ailleurs comparer cet exemple à celui, moins étudié, d'une ataxie cérébelleuse autosomique dominante qui se montre plus sévère et d'apparition plus précoce chez les descendants des pères atteints.

Enfin, le dernier cas, sans doute le plus frappant, est celui de la dystrophie myotonique ou maladie de Steinert (chromosome I9). Transmise par le père, c'est une affection d'évolution lente ne se révélant généralement pas avant l'adolescence. A l'opposé, un enfant né d'une mère atteinte risque de présenter à la naissance un tableau d'hypotonie généralisée qui met sa vie en danger dès les premières heures $[4,5]$.

On a cherché à expliquer ces différences, notamment dans le dernier cas, par l'environnement maternel au cours de la grossesse, ou par la transmission maternelle d'éléments extrachromosomiques, cytoplasme ou mitochondries. Aucune preuve toutef ois n'est venue étayer ces hypothèses. En revanche, l'hypothèse chromosomique a pu être démontrée dans une maladie de la souris, la mutation $\mathrm{T}^{\mathrm{Hp}}$. Celle-ci, qui se reconnaît au raccourcissement de la queue chez les animaux atteints est due à une petite délétion de la partie proximale du chromosome i7; elle n'est apparemment transmise que par les mâles; en fait, elle est léthale lorsque c'est la mère qui en est porteuse. Des expériences de transplantation au stade unicellulaire ont été pratiquées avec des noyaux ou du cytoplasme provenant de femelles porteuses de la mutation $T^{\mathbf{H}_{\mathrm{p}}}$ : elles ont formellement démontré que la propriété "léthale " est le fait du noyau seul, et que le chromosome 17 des souris mâles et femelles fonctionne donc de façon différente [6]. Rappelons pour mémoire que dans tous les exemples mentionnés ici il n'y a aucun écart de gravité de la maladie selon le sexe chez les descendants eux-mêmes.

J.-C.D.

3. Lazzarini A, Mc Cormack MK, Lepore F. Maternal transmission of Huntington disease 62S.

4. Zellweger H, Ionasescu V. Early onset of 1973; 125: 601-4

5. Aicardi A, Conti D, Goutières F. Les formes néonatales de la dystrophie myotonique de Steinert. 7 Neurol Sci 1974; 22: 149-64.

6. Mc Graw J, Solter D. Maternal $T^{H_{p}}$ lethality in the mouse is a nuclear, not cytoplasmic, defect. Nature 1984; 308: 550-I.

I. Erickson RP. Chromosomal imprinting and 